Internist 2012 · 53:1269-1270

DOI 10.1007/s00108-011-2993-2

Online publiziert: 7. Oktober 2012

(c) Springer-Verlag Berlin Heidelberg 2012

U. Hegerl ${ }^{1}$ P. Heußner ${ }^{2} \cdot$ W. Hiddemann ${ }^{2}$

${ }^{1}$ Klinik für Psychiatrie und Psychotherapie, Universitätsklinikum Leipzig

${ }^{2}$ Medizinische Klinik und Poliklinik III, Campus Großhadern, Klinikum der Universität München

\title{
Psychische Störungen in der Inneren Medizin
}

Die Zahl der Patienten, die aufgrund einer Depression oder anderer psychiatrischer Erkrankungen vorübergehend oder dauerhaft arbeitsunfähig werden und zum Teil frühberentet werden müssen, hat in den vergangenen Jahren deutlich zugenommen. Dieser Trend, der aus den Statistiken der Krankenkassen und Rentenversicherungsträger hervorgeht, ist auf den ersten Blick besorgniserregend.

Zumindest teilweise dürfte dies jedoch auch Ausdruck der sehr wünschenswerten Entwicklung sein, dass mehr Erkrankte professionelle Hilfe suchen und dass Ärzte psychiatrische Erkrankungen besser erkennen, behandeln und, vermutlich am wichtigsten, korrekter benennen. Früher wurden z. B. Depressionen öfter hinter weniger negativ besetzten Ausweichdiagnosen wie:

- chronischem Rückenschmerz,

- Tinnitus,

- Fibromyalgie,

- Kopfschmerz und

- "chronic fatigue“

versteckt. Mit „Burnout“ ist allerdings auch eine neue Ausweichdiagnose in Mode gekommen.

Psychische Erkrankungen können dazu führen, dass körperliche Beschwerden, die zuvor gut toleriert wurden, als unerträglich erlebt werden. Umgekehrt können internistische Erkrankungen als Auslöser für psychiatrische Erkrankungen fungieren. Aus diesem Grund ist es erforderlich, dass jeder internistisch tätige Arzt ausreichende Kenntnisse besitzt, um psychische Störungen zu erkennen und gegebenenfalls entsprechende Behandlungsmaßnahmen zu ergreifen. Nicht immer ist allerdings die Grenze zwischen normalen psychologischen Reaktionen, z. B. auf schwere internistische Erkrankungen, und behandlungsbedürftigen psychiatrischen Erkrankungen leicht zu ziehen. Auch die Abgrenzung des sog. Burnout-Syndroms von klar definierten psychiatrischen Erkrankungen erfordert oft psychiatrische Expertise.

\section{) Jeder internistisch tätige Arzt sollte psychische Störungen erkennen können}

Das Ziel unseres Schwerpunkthefts ist es daher, einen aktuellen Überblick über Anpassungsstörungen und einige psychische Erkrankungen zu geben und Eckpunkte der Symptomatik und Behandlung aufzuzeigen. Dazu haben die Autoren zentrale Themenkomplexe übersichtlich dargestellt, die unterschiedliche Aspekte seelischer Anpassungsstörungen und psychischer Erkrankungen in der Inneren Medizin abdecken. So befasst sich der erste Beitrag von M. Strauß et al. mit der Problematik, die Symptomvariabilität von Anpassungsstörungen im Rahmen internistischer Erkrankungen darzustellen und die Abgrenzung gegenüber einer relevanten psychischen Erkrankung zu ermöglichen.

Der Beitrag von H.P. Kapfhammer widmet sich ausführlich der BurnoutProblematik und insbesondere der Frage, $\mathrm{ob}$ es sich dabei um eine eigenständige Erkrankung oder lediglich um ein Symptom handelt. Auch wird die Frage behandelt, wie ein Burnout-Syndrom von einer echten Depression im Sinne einer psychischpsychiatrischen Erkrankung abgegrenzt werden kann. 
$\mathrm{Zu}$ den häufigsten Formen psychischer Störungen gehören Angststörungen, die im Beitrag von F. Geiser et al. auf prägnante Weise dargestellt werden.

Der Beitrag von P. Heußner und $W$. Hiddemann widmet sich dem speziellen Problem seelischer Störungen im Rahmen von Krebserkrankungen. Die Autoren umreißen das Spektrum psychoonkologischer Symptome und psychischer Komorbiditäten sowie deren Behandlung.

Der abschließende Beitrag von $J$. Cordes et al. fasst sehr übersichtlich den Einsatz von Psychopharmaka zusammen und zeigt insbesondere potenzielle Nebenwirkungen und Interaktionen bei Patienten mit kardiovaskulären Erkrankungen auf.

Wir hoffen, mit dem vorliegenden Schwerpunktheft von Der Internist einen aktuellen Überblick über das Spektrum psychischer Erkrankungen in der Inneren Medizin geben zu können und damit dem praktisch tätigen Internisten ein wichtiges Werkzeug für die tägliche Praxis an die Hand zu geben.

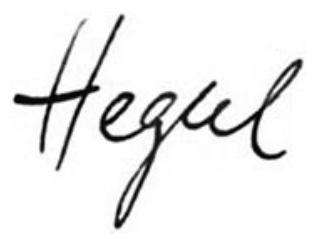

U. Hegerl

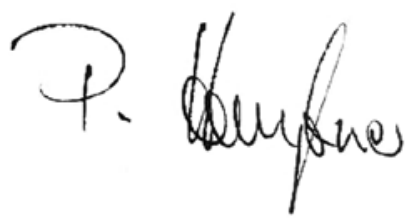

P. Heußner

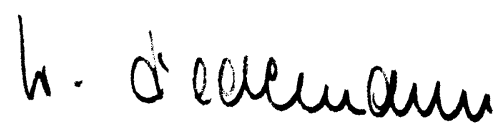

W. Hiddemann

\section{Korrespondenzadresse}

Prof. Dr. W. Hiddemann

Medizinische Klinik und Poliklinik III, Campus Großhadern, Klinikum der Universität München Marchioninistr. 15, 81377 München wolfgang.hiddemann@med.uni-muenchen.de
Harald Salfellner (Hg.) Vitalis-Medizinkalender 2013 Zur Geschichte der Medizin

Prag: Vitalis Verlag 2012, 54 S., (ISBN 978-3-89919-059-5),

Farbe auf Bilderdruckpapier, 29.00 EUR

Der Vitalis-Kalender 2013 „Zur Geschichte der Medizin" führt in die Welt der Heilkunst und Pharmazie. Konzipiert als Wandkalender zum Abreißen bietet er dem Leser bzw. Betrachter unterhaltsame Kurzinformationen zu 53 herausragenden Medizinern und wichtigen Ereignissen der Medizingeschichte.

Jede Woche wird eine neue Mediziner-Persönlichkeit im Kurzporträt vorgestellt. Dazu gehören Berühmtheiten wie der „KnochenBöhler" (Lorenz Böhler), dessen Methode der Frakturbehandlung wegweisend wurde, oder der japanische „Bakterienjäger" Kitasato Shibasaburo, dem es als erstem gelang, Clostridium tetani in Reinkultur zu züchten. Natürlich darf bei einer derart illustren Zusammenschau auch „Der Mann mit dem Durchblick", Wilhelm Conrad Röntgen, nicht fehlen, ebenso wenig wie der deutsche Philosoph und Psychiater Karl Jaspers, der sich besonders für existenzielle Grenzerfahrungen des Menschen interessierte.

Friedensnobelpreisträger Albert Schweitzer, der vor etwa 100 Jahren zusammen mit seiner Frau im damaligen Lambarene ein Urwaldspital gründete und dort Kranke versorgte, ist - in einem Einbaum stehend - auf afrikanischem Gewässer zu sehen. Und eine Fotoaufnahme mit japanischer Bergkulisse erinnert an den berühmten deutschen Bakteriologen Robert Koch und seine zweite Frau Hedwig.

Die Entdeckung der künstlichen Niere - ins

Bild gesetzt durch eine technische Zeichnung des ersten funktionstüchtigen Gerätes zur Blutreinigung - wird ebenso thematisiert wie die Entwicklung der Computertomographie. Knochenreste veranschaulichen die Ausgrabung des Homo floresiensis in Indonesien. Überraschend für den unvorbelasteten Blick: Nicht immer führte die Heilkunst der damaligen Zeit zur Heilung nach heutigem Medizin-Verständnis, so belegt durch die bildhafte Darstellung einer Hinrichtung mit der Guillotine, deren Erfinder der Arzt JosephIgnace Guillotin war.

Zahlreiche Abbildungen von Ärzten, Geräten, anatomischen Schnitten und Büchern sowie
Zitate aus historischen Quellen schmücken die Seiten dieses Kalenders, der eigentlich viel mehr ist als "nur" ein Kalender. Die sorgfältig recherchierten und hochwertig aufbereiteten Informationen sind kurzweiliger Lesestoff auch für zwischendurch.

Fazit: Der Kalender ist fast zu schade, um ihn nur an die Wand zu hängen. Ein ideales Weihnachtsgeschenk für Mediziner und Laien!

Weitere Informationen und Bestellung über www.vitalis-verlag.com

Christiane Weseloh (Heidelberg) 